\title{
Modeling microbially-mediated biogeochemical dynamics in porous media under various flow conditions
}

\author{
HeEwon Jung ${ }^{1}$, Christof MeILE ${ }^{1}$ \\ ${ }^{1}$ Department of Marine Sciences, University of Georgia, \\ Athens, GA 30602, USA, heewon.jung@uga.edu; \\ cmeile@uga.edu
}

Biogeochemical reactions in natural porous media such as soil, sediments, or aquifers, determine global elemental cycling and the fate of contaminants. Microorganisms are of central importance as they mediate many near-surface geochemical processes. However, quantitatively describing microbial processes is a daunting task because of the challenge to resolve the scales relevant to microbial activity, and because of dynamic interactions between microbial communities and their surroundings. Here, we present our efforts to describe complex microbial dynamics within a range of environmentally relevant conditions using reactive transport modeling.

We studied the variability in macroscopic rate estimates under heterogeneous microbial distribution conditions [1]. Pore scale reactive transport analysis revealed that the macroscopic rate estimates are largely determined by flow conditions and reaction kinetics, and to a lesser extent by the distribution of microbial aggregates. We further evaluated physicochemical conditions effectively coordinating microbial phenotype expression mediated by signaling molecules [2]. Modeling results show that advection dilutes signaling molecules so that faster flow conditions require higher microbial densities, faster signal production rates, or higher sensitivities for effective communication through signaling molecules. As a consequence, the induction of quorum sensing is no longer a simple function of cell densities.

This study provides a reactive transport framework to better understand microbial dynamics in various environmental conditions. Ongoing efforts include the development of improved descriptions of microbial metabolism and accounting for inter-species interactions that are emerging from the analysis of omics data.

[1] Jung, H. and Meile, C., 2019. Upscaling of microbially driven first-order reactions in heterogeneous porous media. J. Contam. Hydrol., 224:103483.

[2] Jung, H. and Meile, C., 2020. Mathematical investigation of microbial quorum sensing under various flow conditions. bioRxiv. doi:10.1101/2020.01.09.900027 